\title{
Adaptive Medical Ultrasound Imaging with Data Dependent Weighting Spatial Smoothing Technique
}

\author{
Lutao Wang $^{1, *}$, Jin Gang ${ }^{2}$ and Wei Wang ${ }^{2}$ \\ ${ }^{1}$ School of Computer Science, Chengdu University of Information Technology, Chengdu, Sichuan , 610225, China, \\ ${ }^{2}$ China Aerodynamics Research \& Development Center, Mianyang,Sichuan, 621000, China \\ ${ }^{*}$ Corresponding author
}

\begin{abstract}
In adaptive medical ultrasound imaging, the performance of the adaptive beamformer directly depends on the estimation of the spatial characteristic of the noise and interferences. Accurate estimation of the array covariance matrix leads to significant improvement in image quality compared with non-adaptive delay-and-sum (DAS) beamformers. Most of the techniques have been employed to get a good estimation of array covariance matrix are based on spatial smoothing and diagonal loading. In this paper, spatial smoothing with data dependent weighting has been applied for array covariance matrix estimation, which is then employed in minimum variance (MV) weights calculation. The adaptive weighting spatial smoothing (AWSS) MV beamformer utilizes forward-backward averaging and data dependent weights to make the estimated array covariance matrix as close to Toeplitz matrix as possible. Using AWSS beamformer instead of the normal forward-only beamformers leads to more accurate estimation of the array covariance matrix, significantly improve the imaging resolution and contrast without the need for temporal smoothing and diagonal loading. The performance of the proposed approach is demonstrated by several simulated examples.
\end{abstract}

Keywords-ultrasound imaging; adaptive beamforming; spatial smoothing; minimum variance

\section{INTRODUCTION}

In medical ultrasound imaging, reducing the destructive contribution of the off-axis echoes, originating from off-axis targets, can result in images with enhanced resolution and contrast. The off-axis echoes elimination capabilities of image array depends on the number of array elements and their weights. Delay-and-sum (DAS) beamforming is the standard technique in medical ultrasound imaging. As predetermined weights are used to construct the echo signal originating from the main target, the beamformed signals have a wide the mainlobe and large sidelobe levels. This means DAS beamformer has poor resolution and weak suppression of interference signals. The sidelobe of the DAS beamformer can be controlled using aperture shading, but this increases the mainlobe width. In other word, applying DAS beamformer in medical ultrasound imaging results in enhanced contrast at the expense of resolution. In contrast to DAS beamformer,

Adaptive beamforming technique uses the estimation of spatial characteristics of noise and interferences to calculate the aperture weight vector. The weights of adaptive beamformers are data-dependent and time-varying. Thus, it can suppress off-axis echoes effectively and lead to simultaneous improvement in resolution and contrast [1].
Adaptive beamformer have been applied to medical ultrasound imaging by several researchers, and demonstrated improved imaging contrast and resolution compared with DAS beamformer in the past years [2]. Mann and Wallker [3] first applied a constrained adaptive beamformer on experimental data of a single-point and a cyst phantom, showing improved resolution and contrast. Synnevag et al. [4] applied adaptive beamformer on both simulated and experimental data and showed that adaptive beamformer outperforms DAS in terms of contrast and resolution. Recently, the combination of the $\mathrm{MV}$ beamformer and coherence factor (CF) weighting has been applied to ultrasound data and demonstrated the effectiveness in simultaneous improvement of imaging resolution and contrast [5].

Most of the above adaptive beamformers are based on the minimum variance (MV) beamforming theory devised by Capon. The weights of MV beamformer are computed by minimizing the power of beamformer outputs subject to the constraint that the beamformer must gives a constant response in the look-direction. Under the assumption that the signals of interest must not be correlated with the noise and interference and sufficient statistical information is available to form a robust covariance matrix, the weights of MV beamformer are optimum. Unfortunately, these constrains are not satisfied in medical ultrasound imaging and several measures must be taken to enhance the image quality. Thus, spatial smoothing, diagonal loading and temporal averaging are combined to obtain an accurate estimation of the array covariance matrix before applying MV method on returned echoes [6]. In [7], spatial smoothing (SS) was applied to medical ultrasound imaging to decorrelate the strongly correlated returned echoes and get a more robust estimation of array covariance matrix. In [8], diagonal loading (DL) and temporal smoothing techniques were employed to make the MV method be robust to parameter mismatch and have speckle that resembles that of conventional imaging.

In this paper, we apply adaptive spatial smoothing beamformer to medical ultrasound imaging. By simulation examples, we show that our proposed method properly satisfied with the medical ultrasound limitations and results in simultaneously improvement of imaging resolution, contrast and sidelobes, outperforms both MV and DAS beamformers. We use adaptive weighting spatial smoothing (AWSS), instead of the conventional forward only (F-only) spatial smoothing to obtain a more accurate estimation of array covariance matrix, which is needed for computing the optimum weights of MV 
beamformer. In addition, we show that when we use AFB weights instead of MV weights, the signal cancellation can be effectively mitigated and the proposed beamformer is less depend on the choice of model parameters, such as the amount of diagonal loading, the number of samples needed for temporal averaging.

The rest of the paper is organized as follows. Section 2 contains the sensor signal model and presents the MV solution. Our proposed method is represented in Section 3. The performance evaluation of our proposed method compared with DAS beamformer and MV beamformer on simulated data are illustrated in Section 4. Finally, we draw conclusion in Section 5.

\section{SNESOR SIGNAL AND MV-BEAMFORMING}

Consider a uniformly spaced linear array of $\mathrm{M}$ elements and some point scatters in the near-field of the array. The vector of array observations vector at time $t$ could be modeled as

$$
\mathbf{x}(t)=\mathbf{s}(t) \mathbf{a}+\mathbf{i}(t) \mathbf{a}_{I}+\mathbf{n}(t)
$$

where $\mathbf{s}(t)$ is the desired signal, $\mathbf{i}(t)$ and $\mathbf{n}(t)$ are off-axis interference and noise components received by array, respectively. $\mathbf{a}$ and $\mathbf{a}_{I}$ is the steering vector of the desired signal and interferences with wavelength $\lambda$ arriving from angle $\theta$, respectively:

$$
\mathbf{a}=\left[1, e^{-j 2 \pi \sin (\theta) d / \lambda} \cdots e^{-j 2 \pi \sin (\theta) d(M-1) / \lambda}\right]^{\mathrm{T}}
$$

where $d$ represent the space of array element.

The output of a beamformer for time $t$ is the weighted combination of the individual channel data:

$$
y(t)=\mathbf{w}^{\mathrm{H}} \mathbf{x}(t)=\sum_{i=1}^{M} w_{i}(t) x_{i}\left(t-\Delta_{i}\right)
$$

where $w_{i}(t)$ is the weight for array element $i$, and $\mathbf{w}=\left[w_{1}, \cdots, w_{M}\right]^{\mathrm{T}} \in \mathrm{C}^{M \times 1} . \Delta_{i}$ is the time delay applied to element $i$ to focus at a special point in the image, and $(\cdot)^{\mathrm{T}}$, $(\cdot)^{\mathrm{H}}$ denotes transpose and conjugate transpose respectively.

The classical MV beamformer minimizes the array output energy while maintaining a distortionless response to the desired signal

$$
\min _{\mathbf{w}} \mathbf{w}^{\mathrm{H}} \mathbf{R w} \quad \text { s.t. } \quad \mathbf{w}^{\mathrm{H}} \mathbf{a}=1
$$

The solution to (4), which can be easily solved by means of using Largrange multiplier method, is given by

$$
\mathbf{w}=\frac{\mathbf{R}^{-1} \mathbf{a}}{\mathbf{a}^{\mathrm{H}} \mathbf{R}^{-1} \mathbf{a}}
$$

In practice, it is not feasible to calculate the exact spatial covariance matrix $\mathbf{R}$ and it would be estimated by the sample covariance matrix

$$
\hat{\mathbf{R}}=\frac{1}{K} \sum_{k=1}^{K} \mathbf{x}(k) \mathbf{x}^{H}(k)
$$

where $K$ is the number of recently received samples.

\section{ESTIMATION OF SPATIAL COVARIANCE MATRIX}

As mentioned earlier, the weights of MV beamformer are calculated from the estimation of spatial covariance matrix. Thus, in adaptive ultrasound imaging, the performance of the adaptive beamformer, and consequently the quality of the ultrasound image, directly depends on the accurate estimation of the array covariance matrix. To get a more accurate estimation of array covariance matrix, several methods need to taken before applying MV beamformer on medical ultrasound imaging.

SS is the main measure used to decorrelate the high coherence between the on-axis and off-axis echoes, which may result in signal cancellation. In this method, the $M$ elements linear array is grouped into $M-L+1$ subarrays of length $L$, each overlapping its neighboring subarray by all but one element:

$$
\begin{aligned}
\mathbf{x}_{l}(t) & =\left[x_{l}\left(n-\Delta_{l}(n)\right), \ldots, x_{l+L-1}\left(n-\Delta_{l+L-1}(n)\right)\right]^{\mathrm{T}} \\
l & =0,1, \ldots, M-L
\end{aligned}
$$

The covariance matrix of these subarray are averaged to estimate the spatially smoothed array output covariance matrix

$$
\begin{aligned}
\hat{\mathbf{R}}_{S}(t) & =\frac{1}{M-L+1} \sum_{l=1}^{M-L+1} \mathbf{x}_{l}(t) \mathbf{x}_{l}{ }^{H}(t) \\
& =\frac{1}{M-L+1} \sum_{l=1}^{M-L+1} R_{l}(t)
\end{aligned}
$$

Temporal averaging means averaging over a small range gate, which is comparable in extent to the transmit pulse. By using temporal averaging, similar speckles statistics that resembles DAS speckle statistics can be retained. The general estimate of the covariance matrix with subarry of length $L$ and temporal averaging over $2 K+1$ samples then becomes

$$
\hat{\mathbf{R}}_{S T}(t)=\frac{1}{(2 K+1)(M-L+1)} \sum_{n=-K}^{K} \sum_{l=1}^{M-L+1} \mathbf{x}_{l}(t+n) \mathbf{x}_{l}{ }^{H}(t+n)
$$

The transmitted pulses in medical ultrasound imaging are short and nonstationary. Hence, the samples that can be used to calculation spatial covariance matrix estimation are limited. To estimate the spatial covariance matrix more accurately and improve the robustness of the MV beamformer against model mismatch and numerical errors, the spatial covariance matrix is diagonally loaded by adding a scaled identity matrix. After applying (7), (9) and DL to estimate the spatial covariance matrix, the $L \times 1 \mathrm{MV}$ optimum weights can be rewritten as

$$
\hat{\mathbf{w}}=\frac{\left(\mathbf{R}_{S T}+\gamma \mathbf{I}\right)^{-1} \mathbf{a}}{\mathbf{a}^{\mathrm{H}}\left(\mathbf{R}_{S T}+\gamma \mathbf{I}\right)^{-1} \mathbf{a}}
$$

where $\gamma=\Delta \cdot \operatorname{trace}\left(\hat{\mathbf{R}}_{S T}(t)\right)$ is the loading factor, which can be set to $\Delta$ times of the power of the received signals [6]. 


\section{SPATIAL Covariance Matrix WITH AdAPTIVE WEIGHTS}

SS is a kind of matrix tapering method which highlights the diagonal elements of the spatial covariance matrix. Thus, SS cannot avoid signal cancellation owing to the remaining correlation between the on-axis signals and off-axis signals for finite array size. Besides, the interference rejection ability of SS depends on the spacing and the directions of the coherent sources. In the case of small-size array and closely-spaced coherent signals, SS always proves futile.

To improve the interference rejection performance and eliminate signal cancellation, we use AWSS method instead of SS to estimate the spatial covariance matrix. In this approach, the spatially averaged covariance matrix $\hat{\mathbf{R}}_{W}$ is given by

$$
\hat{\mathbf{R}}_{W}(t)=\frac{1}{M-L+1} \sum_{l=1}^{M-L+1} v_{l} R_{l}(t)
$$

where $\mathbf{V}=\left[v_{1}, \ldots v_{L-M+1}\right]$ is averaging weights vector for the spatial covariance matrix of the subarry. The proper choice of $\mathbf{V}$ can be considered to be a linearly constrained minimization and it can be expressed as follows:

$$
\min _{\mathbf{v}}|\rho|^{2} \mathbf{V} \mathbf{a}_{I} \mathbf{a}_{I}^{\mathrm{H}} \mathbf{V}^{\mathrm{H}} \quad \text { s.t. } \quad \mathrm{V}^{\mathrm{H}} \mathbf{E}=1
$$

where $\rho$ is the correlation factor, $\mathbf{E}$ is a $L$-dimensional vector in which all the elements are unity. The solution to (12) is

$$
\mathbf{V}_{\text {opt }}=\frac{\mathbf{D}^{-1} \mathbf{E}}{\mathbf{E}^{\mathrm{H}} \mathbf{D}^{-1} \mathbf{E}}
$$

where $\mathbf{D}=\mathbf{a}_{I} \mathbf{a}_{I}^{\mathrm{H}}$. (13) is valid for any angle of interference arrival and any subarray size greater than two. Thus, applying $\mathbf{V}_{\text {opt }}$ in spatial covariance matrix estimation ensures complete decorrelation of the on-axis signals and off-axis interference regardless of the arrival angle of the interference and the size of the subarray. As the arrival angles of interference are often unknown, the matrix $\mathbf{D}$ can be replaced by the known subarray matrix $\hat{\mathbf{R}}_{S}$

In [16], Asl et al. applied MV beamformer coupled with forward-backword spatial smoothing to decorrelate on-axis and off-axis signals and also get a good estimate of the spatial covariance matrix. Based on the theory of forward-backword spatial smoothing, the subarry spatial covariance $R_{l}(t)$ matrix in (8) can be replace by

$$
R_{F B}^{l}(t)=\frac{1}{2}\left(R_{l}(t)+\mathbf{J} R_{l}^{\mathrm{T}}(t) \mathbf{J}\right)
$$

where $\mathbf{J}$ is the reflection matrix that has unity elements along the cross diagonal. Then, the final smoothed spatial covariance matrix is given by

$$
\hat{\mathbf{R}}_{W}(t)=\frac{1}{2(M-L+1)} \sum_{l=1}^{M-L+1} v_{l}\left(R_{l}(t)+\mathbf{J} R_{l}^{\mathrm{T}}(t) \mathbf{J}\right)
$$

By estimation of the subarry spatial covariance matrix $R_{l}(t)$, the optimal weighting vector, $\mathbf{w}=\left[w_{o}^{1}, \cdots, w_{o}^{L}\right]^{\mathrm{T}} \in \mathrm{C}^{L \times 1}$, can be computed by (15), (5), and then the output of the proposed beamformer can be given by

$$
y(t)=\frac{1}{M-L+1} \sum_{l=1}^{M-L+1} w_{o}^{l} R_{l}(t)
$$

\section{SiMULATION AND RESULTS}

In this section, we provide several simulation examples to show that the proposed beamformer outperforms DAS beamformer and MV beamformer in terms of resolution, contrast and robustness.

In all examples, we applied fixed focus on transmission and dynamic focus on reception. By doing so, the echoes originating from receiving focus seemingly always arrives from broadside. Thus, the steering vector of MV beamformer simply becomes a vector of ones. For MV beamformer, spatial smoothing with subarray length of $L=M / 2$ was used to decorrelate the on-axis and off-axis echoes and mitigate signal cancellation. Then, the estimated spatial covariance matrix was diagonally loaded to increased numerical stability when computing the optimum weights. Temporal averaging over $2 K+1$ samples was also implemented to improve the robustness of the MV beamformer. For the proposed beamformer, the spatial covariance matrix was estimated by (15) and the optimum weighting vectors were computed by (5) before beamforming.

All the simulations were performed with a ULA consisting of 64 elements with $\lambda / 2$ spacing. using Field II [9]. The central frequency of the array elements was set to $2.5 \mathrm{MHz}$. The excitation pulse was modeled as a two-cycle square at the central frequency of the array, and the bandwidth was $60 \%$. The speed of propagation was $1540 \mathrm{~m} / \mathrm{s}$.

\section{A. Simulated Point Targets}

Figure 1 shows the reconstructed B-mode images of scatterer pairs over $60 \mathrm{~dB}$ display dynamic range, with rectangular, MV, and the proposed beamformers. The 10 simulated point scatters were located at every $20 \mathrm{~mm}$ from 40 $\mathrm{mm}$ and separated by $2 \mathrm{~mm}$. 


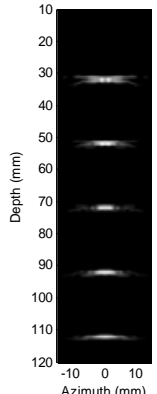

(A)

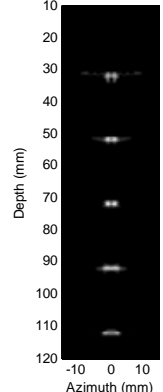

(B)

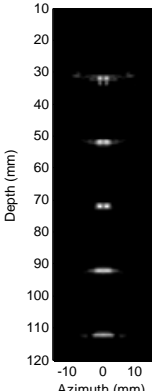

(C)

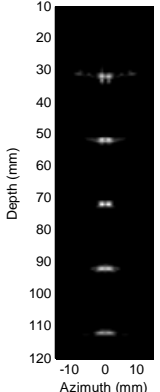

(D)
FIGURE I. SIMULATED POINT TARGETS: (A) DAS (B) MV (No DL) (C) MV (DL $=1 / 100 \mathrm{~L}, \mathrm{~K}=20)(\mathrm{D})$ AWSS-MV

As shown in Figure 1, the rectangular weighting DAS beamformer has poor lateral resolution and high sidelobes, it can hardly resolve the scatterers and presents the worst performance. By effectively suppressing off-axis signals and reducing the sidelobes, The MV beamformer in Figure 1 (b) leads to much better lateral resolution than the DAS. But it can not accurately estimates the magnitudes of the scatterer pair located at $30 \mathrm{~mm}$. The beamforer employing DL ( $\Delta=1 / 100 L)$ and temporal averaging ( $K=10$ ) can results in more accurate estimation of the scatterer pairs at the expanse of reduced resolution and contrast. These results were presented in Figure 1 (c). Figure 1 (d) shows that the beamformer using the proposed spatial covariance matrix estimation approach can accurately estimate the amplitude of the scatterer pairs and presents the better lateral resolution for the scatterer pairs.

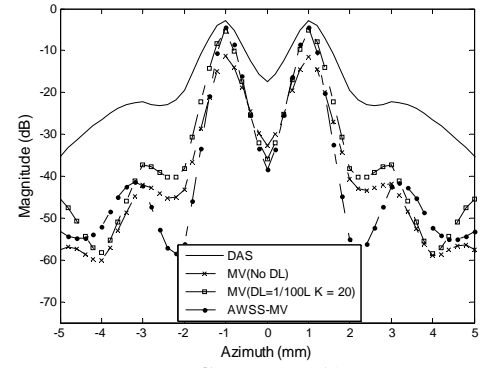

FIGURE II. (A)

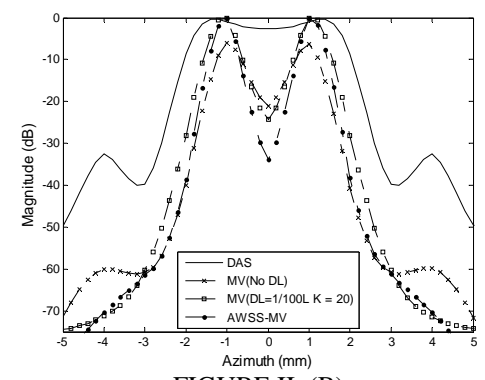

FIGURE II. (B)

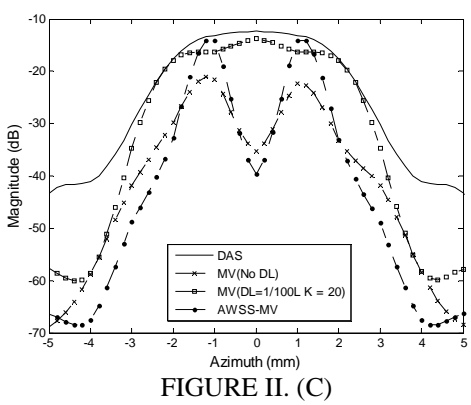

FIGURE II. SIMULATED LATERAL CROSS SECTIONS: (A) 3MM (B) $70 \mathrm{MM}(\mathrm{C}) 90 \mathrm{MM}$

In Figure 2 we show the lateral variation of the beamformed responses located at depths of $30 \mathrm{~mm}, 70 \mathrm{~mm}$, and $90 \mathrm{~mm}$, respectively. As results show, the proposed beamformer presents accurate estimation of amplitude of the scatterer pairs at all depths. The amplitudes of the point targets were underestimated by about $9 \mathrm{~dB}$ for $\mathrm{MV}$ beamformer. Adding DL and temporal averaging to MV beamformer leads to much accurate estimation of the spatial covariance matrix, which can effectively compensate the amplitude loss. But this leads to broaden mainlobe width compared to MV (No DL) beamformer. It also can be seen that the proposed beamformer presents the narrowest mainlobe and lowest sidelobes. Figure 1 (b) shows that the scatterer pairs were better resolved in the proposed beamformer by about $3 \mathrm{~dB}$ and $12 \mathrm{~dB}$ comparing with MV (No DL) and $\mathrm{MV}(\Delta=1 / 100 L, K=10)$, respectively. In Figure 1 (c), we see that the DAS and MV ( $\Delta=1 / 100 L, K=10$ ) beamformer are unable to resolve the scatterer pairs while the proposed beamformer can resolve them by up to $40 \mathrm{~dB}$. The sidelobe of the proposed beamformer are about $15 \mathrm{~dB}$ lower than DAS ones and $6 \mathrm{~dB}$ lower than MV (No DL) ones.

\section{B. Simulated Cyst Phantom}

To investigate the contrast of the beamformers, a circle cyst phantom in a speckle pattern was simulated. The circular cyst has a radius of $3 \mathrm{~mm}$ and center at $(x, z)=(0,50) \mathrm{mm}$. The speckle pattern was simulated with 10 randomly placed scatters within a resolution cell of $\lambda^{3}$ to ensure fully developed speckle [10], where $\lambda$ was the central wavelength of the propagating waveform in the medium. The scattering amplitudes were Gaussian distributed. The resultant images were shown in Figure 3. All images are displayed with $60 \mathrm{~dB}$ dynamic range.

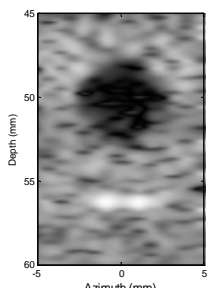

(A)

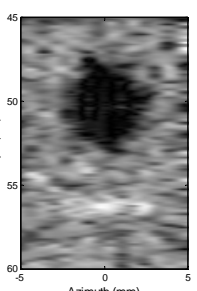

(B)

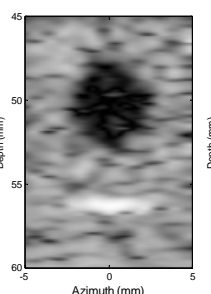

(C)

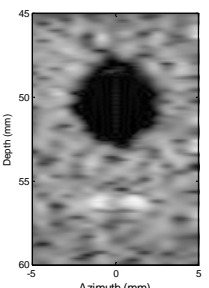

(D)
FIGURE III. SIMULATED CYST PHANTOM. (A) DAS (B) MV (NO DL) $\begin{array}{ll}\text { (C) MV-DL } & \text { (D) AWSS-MV }\end{array}$ 


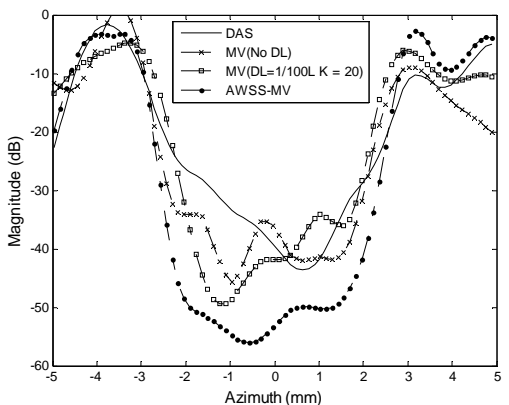

FIGURE IV. CROSS SECTIONS OF SIMULATED CIRCULAR CYST

The cross-section for the tested cyst was shown in Figure 4. It is clear that the proposed method demonstrates superior contrast than DAS and MV beamformers. We also see that the noise inside the cyst is suppressed better by the proposed beamformer than that of DAS and MV beamformers. It also can be seen that the decrease of the proposed beamformer to the background noise level is faster than the others.

We further confirmed the superiority of the proposed beamformer in terms of contrast ratio (CR) and the contrast to noise ratio (CNR). The CR is defined as the ratio of the mean value in the background to the mean value in the cyst region, and the CNR is defined as the CR divided by the standard deviation of image intensity in the background region. Table. 1 lists the relative CR and CNR for each beamforming method.

\section{TABLE I. CONTRAST STATICS}

\begin{tabular}{|l|l|l|l|l|l|}
\hline $\begin{array}{l}\text { Weighting } \\
\text { Methods }\end{array}$ & $\begin{array}{l}\text { Mean } \\
\text { intensity in } \\
\text { cyst region } \\
(\mathrm{dB})\end{array}$ & $\begin{array}{l}\text { Mean intensity } \\
\text { in background } \\
(\mathrm{dB})\end{array}$ & $\begin{array}{l}\mathrm{CR} \\
(\mathrm{dB})\end{array}$ & $\begin{array}{l}\mathrm{CN} \\
\mathrm{R}(\mathrm{dB})\end{array}$ & $\begin{array}{l}\text { Background } \\
\text { standard } \\
\text { deviation } \\
(\mathrm{dB})\end{array}$ \\
\hline DAS & -49.85 & -25.45 & 24.40 & 3.52 & 6.92 \\
\hline MV (No DL) & -53.65 & -29.42 & 24.23 & 3.41 & 7.10 \\
\hline MV (DL) & -51.42 & -25.25 & 26.17 & 3.88 & 6.75 \\
\hline AWSS-MV & -59.74 & -25.47 & 34.27 & 4.90 & 7.04 \\
\hline
\end{tabular}

As seen from Tab. 1, the MV (No DL) beamformer presets the worst contrast. The MV beamformer ( $\Delta=1 / 100 L, K=10$ ) gives CR improvement of $1.7 \mathrm{~dB}$ and CNR about $7.5 \%$ compared with DAS beamformer. Also, the background standard deviation is approximately the same for these two beamformers. Using our proposed beamforming method results in an image in which the speckle intensity is similar to DAS while the cyst region intensity is decreased by about 9.9 $\mathrm{dB}$ with respect to DAS. As a result, the CR of the proposed beamformer is increased by about $9.8 \mathrm{~dB}$ compared with DAS. In addition, the proposed beamformer obtains a $5.23 \mathrm{~dB}$ increase of CR and about $25.6 \%$ improvement of CNR relative to MV (No DL) beamformer. Thus, our proposed AWSS-MV beamformer results in significant improvement of contrast, outperforming DAS and MV beamformers .

\section{CONCLUSIONS}

In this paper, we have applied an AWSS-based MV beamformer to medical ultrasound imaging and shown the effectiveness of the proposed beamformer using simulated phantoms. The proposed beamformer uses adaptive weighting spatial smoothing instead of forward only spatial smoothing to obtain a more accurate estimation of the spatial covariance matrix, which is then used to MV weights calculation. This leads to improved sidelobes, amplitude resolution, and less model parameter dependent compared with MV beamformer combined with diagonal loading. Therefore, applying the proposed beamformer to medical ultrasound imaging can significantly enhance the resolution and contrast of the images, outperforming both the DAS and regularized MV beamformers.

\section{REFERENCES}

[1] P . Stoica, Z. Wang, and J. Li, Robust Capon beamforming, IEEE Signal Process. Lett., 10. 6 (2003) 172-175.

[2] J.-F. Synnevag, A. Austeng, and S. Holm, Adaptive beamforming applied to medical ultrasound imaging, IEEE Trans. Ultrason. Ferroelectr.Freq. Control, 54. 8 (2007) 1606-1613.

[3] J. A. Mann and W. F. Walker, A constrained adaptive beamformer for medical ultrasound: Initial results, in Proc. IEEE Ultrasonics Symp., 2002, pp. 1807-1810.

[4] J.-F. Synnevag, A. Austeng, and S. Holm, Benefits of minimumvariance beamforming in medical ultrasound imaging, IEEE Trans.Ultrason. Ferroelectr. Freq. Control, 56. 9 (2009) 1868-1879.

[5] B. Mohammadzadeh-Asl and A. Mahloojifar, Minimum variance beamforming combined with adaptive coherence weighting applied to medical ultrasound imaging, IEEE Trans. Ultrason. Ferroelectr.Freq. Control, 56. 9 (2009) 1923-1931.

[6] B. Mohammadzadeh-Asl and A. Mahloojifar, Eigenspace-based minimum variance beamforming applied to medical ultrasound imaging,IEEE Trans. Ultrason. Ferroelectr. Freq. Control, 57. 11 (2010) 2381-2390.

[7] T. J. Shan, M. Wax, and T. Kailath, On spatial smoothing for direction-of-arrival estimation of coherent signals, IEEE Trans.Acoust. Speech Signal Process., 33. 4 (1985) 806-811.

[8] B. Mohammadzadeh-Asl and A. Mahloojifar, Contrast Enhancement and Robustness Improvement of Adaptive Ultrasound Imaging Using Forward-Backward Minimum Variance Beamforming. IEEE Trans. Ultrason. Ferroelectr. Freq. Control, 58. 4 (2011) 858-867.

[9] J. A. Jensen, Field: A program for simulating ultrasound systems, Med. Biol. Eng. Comput., 34 (1996) 351-354.

[10] J.-F. Synnevag, C. I. Colombo Nilsen, and S. Holm, Speckle statistics in adaptive beamforming, in Proc. IEEE Ultrason. Symp., 2007, pp. 1545-1548. 